\title{
Learning Bayesian Network Structure from Environment and Sensor Planning for Mobile Robot Localization
}

\author{
Hongjun Zhou \\ Chuo University, Tokyo, Japan \\ Email: zhou@indsys.chuo-u.ac.jp
}

\author{
Shigeyuki Sakane \\ Chuo University, Tokyo, Japan \\ Email: sakane@indsys.chuo-u.ac.jp
}

\begin{abstract}
In this paper we propose a novel method of sensor planning for a mobile robot localization problem. We represent causal relation between local sensing results, actions, and belief of the global localization using a Bayesian network. Initially, the structure of the Bayesian network is learned from the complete data of the environment using K2 algorithm combined with GA (genetic algorithm). In the execution phase, when the robot is kidnapped to some place, it plans an optimal sensing action by taking into account the trade-off between the sensing cost and the global localization belief which is obtained by inference in the Bayesian network. We have validated the learning and planning algorithm by simulation experiments in an office environment.
\end{abstract}

\section{INTRODUCTION}

The mobile robot navigation and localization is very traditional and fascinating research theme. Until now, a lot of researches have been focused on how to obtain an accurate map, and then how to match the sensing information of the robot to the map for localization. However, in robot navigation, sensor information is prone to errors and a slight change of the robot's pose deteriorates the sensing results. Therefore, in the past decades, many probabilistic approaches have been proposed to cope with uncertainty and to improve robustness of the localization[1]. However, less work has been done in sensor planning for the localization.

Fox et al.[2] proposed an Active Markov Localization method for improving the efficiency in localization. However, since their system is based on the first order Markov process, it can not represent complex relation between actions, local information, and global localization. Kristensen[3] proposed a mobile robot sensor planning approach based on a topdown decision tree algorithm. However, the utility based Bayesian decision tree theory is too simple to catch the causal relations between local sensing information and global localization. A multiple hypothesis tracking approach has been used in active global localization [4]. However, the Kalman filter based approach must assume model of linear dynamics with Gaussian noise. Zhou et al.[5] proposed an algorithm to reconstruct a $B N$ and use it to plan efficient sensing action for the mobile robot localization. Since the system deals with partial environment information, the planned sensing action may be locally optimal. Moreover, the causal relations of the $B N$ nodes were manually designed.

In this paper, we propose a sensor planning method for mobile robot localization. Initially, we represent causal relations

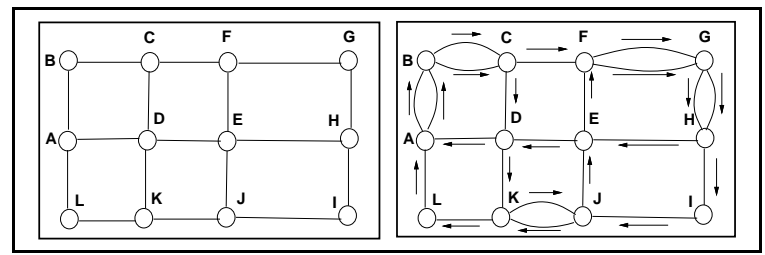

Fig. 1. (left) A graph to represent the topology of the environment. (right) A path (from A to A) obtained as a solution of Chinese postman problem.

between local sensing results, actions, and belief of the global localization in a Bayesian network $(B N)$ structure. The BN structure, as well as the parameters, is learned automatically from the environment data using $\mathrm{K} 2$ algorithm combined with GA (genetic algorithm). In the execution phase, when the robot is kidnapped to some place, it plans an optimal sensing action by taking into account the trade-off between the sensing cost and the global localization belief which is obtained by inference in the $B N[6]$.

\section{ENVIRONMENT INFORMATION GATHERING AND $B N$ CONFIGURATION}

\section{A. Path for Environment Information Gathering}

We performed the simulation experiments in an office environment (Fig. 9). Initially, to obtain complete environment information, the robot must navigate in all of the corridors and intersections. We employ a framework of the Chinese postman problem [7]. The Chinese postman problem requires finding the shortest tour in a graph which visits every edge at least once. As shown in Fig. 1, we represent the topology of the environment as a graph and search a path from $A$ to $A$ using the next node algorithm [8]. Then the robot navigates in all corridors and intersections along the path and gathers the environment information to be used for localization tasks.

\section{B. Environment Representation and BN Configuration}

We define a segment $(S g)$ as the environment information of a corridor between two neighboring intersections. One segment involves four kinds of information as follows:

1) Two intersection labels,

2) Landmarks on both sides of the corridor between two intersections, 


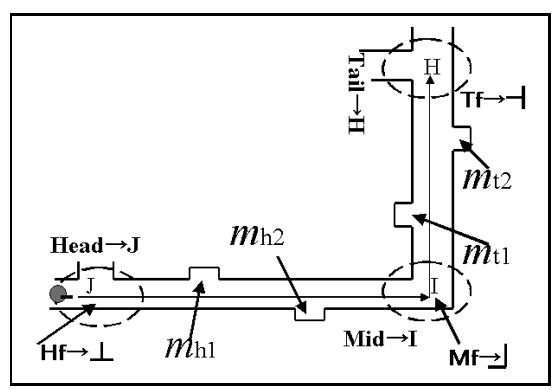

Fig. 2. Mapping the environment information of two neighboring corridors into nodes of $B N$.

3) Geometric features of the intersections sensed when the robot enters the intersections,

4) Action taken by the robot when it enters the corridor.

In our system, we call the environment information of two neighboring corridors an environment information set. The information of every environment information set (for example, label of an intersection, geometrical feature of an intersection, etc.) corresponds to a value of nodes in $B N$.

We define sensing information as observable variables, and labels of intersections as hypothesis variables of a $B N$. We put together all of the environment information of two neighboring corridors and save them into a training database. The database is used to learn the parameters and structure of $B N$. For example, the training database obtained from the environment (Fig. 9) has 138 data cases. The $B N$ (Fig. 4) is learned from the training database. In this case, the $B N$ has 13 probabilistic variables (nodes). As shown in Fig. 2, the nodes, Head, Mid, Tail, are defined by labels of the entrance intersection, middle intersection, and exit intersection of two neighboring corridors, respectively. In the experiments, the nodes Head, Mid, Tail have twelve possible values $(A, B, \ldots, L)$. The nodes Action 1 and Action 2 denote the actions which the robot takes when it enters head and middle intersections, respectively. The action nodes have three possible values: forward, turn left, turn right. The nodes $H f, M f, T f$ correspond to geometric features (such as a range pattern) recognized by the robot when it enters the entrance, middle, and exit intersections, respectively. As shown in Fig. 2, these nodes have six possible values: $+, \top, \dashv$ $, \vdash, \neg$ and so on. In Fig. 2, there are four possible landmarks (hollows) in two neighboring corridors, represented by the nodes $m_{h 1}, m_{h 2}, m_{t 1}, m_{t 2}$. In the experiments, we assume that two hollows can appear on a side of a corridor, and the hollows are used as landmarks. We define the landmark in a list (geometric feature, local distance ${ }^{1}$ ). The landmark nodes have four possible values: " $1 \sim 4$ " which denotes four layout types of the landmark. In addition, we define a mediating variable [6], Cn, by label of every data set, has 138 values.

\section{LEARNING $B N$ STRUCTURE FROM DATA}

$B N$ is a directed acyclic graph that represents dependencies between probabilistic variables. An arc between two nodes

\footnotetext{
${ }^{1}$ The distance between an intersection and its neighboring landmark, or two neighboring landmarks
}

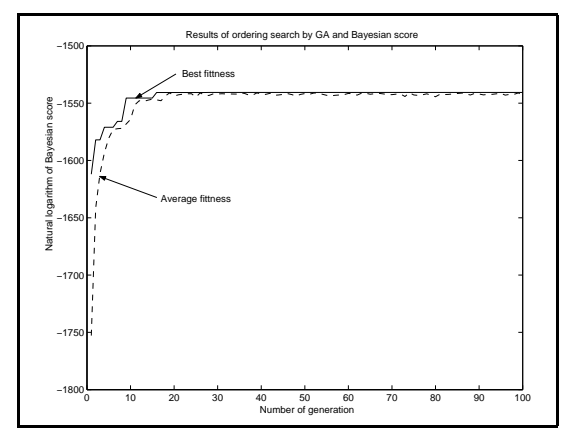

Fig. 3. The results of ordering searching by GA and Bayesian score

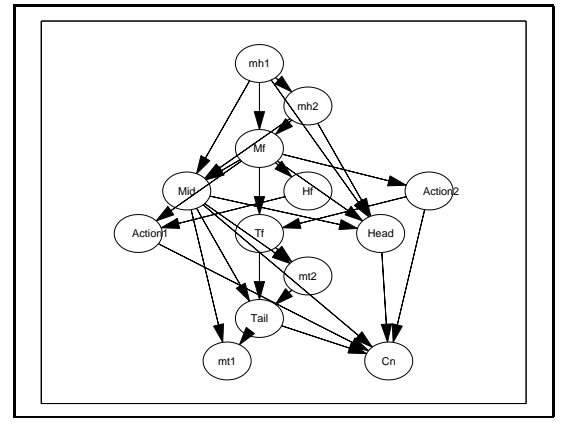

Fig. 4. Learned $B N$ 's structure by $K 2$ and GA

of $B N$ represents the causal relation between the nodes. However, it is often difficult to determine the casual relation among nodes. In our localization tasks, we usually do not know which landmark has dependency with the other nodes, so we take a $B N$ structure learning approach instead of designing the network structure manually.

\section{A. K2 Algorithm Combined with GA}

We apply a structure search method based on Bayesian score, named the $K 2$ algorithm [10], to learn the causal relation between local environment information, robot action, and global localization. The Bayesian score is a joint probability $P\left(B_{s}, D\right)$ between $B N$ structure $\left(B_{s}\right)$ and database $(D)$. The $K 2$ algorithm is a greedy search algorithm. Ref[10] describes that the search space is too huge to evaluate all of the possible structures. To reduce the search space, the $K 2$ algorithm uses a constraint of ordering of nodes (i.e., the causal attributes of a node should appear earlier in the order). However, it is often difficult to determine the order.

In our system, we employ a genetic algorithm(GA) to search the best ordering as described in Ref. [11]. Using this ordering, $K 2$ learns the best $B N$ structure from the data. Then the Bayesian score of $K 2$ gives a fitness value to $G A$. The combination of $G A$ and $K 2$ iterates until the average fitness is improved no further.

\section{B. Example of BN Structure Learning}

Using the training database, we attempt to learn a structure of $B N$. The population size of the $G A$ is 80 and the algorithm uses crossover and mutation operations. Figure 3 shows the 
convergence of fitness value with 100 generations. The dashed line and solid line in the figure show the average and the best fitness scores of each generation, respectively. By combining the $K 2$ algorithm with the $G A$ search, we can obtain a suboptimal ordering of the nodes and a semi-optimal $B N$ structure as shown in Fig. 4.

\section{Sensor Planning for Localization}

\section{A. Summary of the Sensor Planning System}

The execution phase of the planning system consists of the following three steps:

1) Inference for localization: Initially, a mobile robot starts navigation from an unknown position. While the robot is sensing in a corridor, the $B N$ is used to infer the global localization belief whenever the robot obtains new sensing information.

2) Prediction for sensor planning: If the sensing information of this corridor is insufficient for localization, the system predicts possible actions and sensing information to be obtained by the actions. The sensor planner runs at the exit intersection of the sensed corridor.

3) Sensor planning for localization: Then the sensor planner uses the predicted information to select an optimal sensing action to perform active sensing by taking into account of the global localization belief and sensing cost.

\section{B. Inference for Localization}

The robot starts navigation from an unknown position without sensor planning. The navigation basically uses a potential method in a corridor. The robot gathers sensing information events, including landmarks and geometric features of intersections, in the current corridor. Then the information events are given to the $B N$ as evidences to infer global localization, i.e., which corridor the robot has sensed. The probability of the corridor's label is calculated as $P$ (Head, Mid,Tail|obtained sensing event) using the $B N$.

We define belief of the global localization (TolBef) as follows:

$$
\text { TolBef }=(1 / 2) \times(\max (P(\text { Head }))+\max (P(\text { Mid })))
$$

where $\max (P($ Head $))$ and $\max (P(M i d))$ are the maximum values of the probability of node Head and Mid, respectively. $P(\mathrm{Head})$ and $P(\mathrm{Mid})$ are calculated by the $B N$ inference.

If $T o l B e f \geq t h d 1$, the system terminates the localization process. Because in this case the robot can estimate the labels of the corridors only by using the current environment information, there is no need to perform sensor planning. Otherwise (TolBef $<t h d 1$ ), the robot has to move to the next corridor to perform active sensing. Therefore, the sensor planner selects an optimal sensing action for the localization.

Since the $B N$ of our system is not a tree structure but has loops as shown in Fig. 4, we use the Junction tree algorithm [6] to infer probabilities of the nodes.

\section{Prediction for Sensor Planning}

The sensor planner consists of two processes: (1) prediction and (2) planning. The prediction process predicts some possible actions and sensing information expected to be obtained by these actions. The prediction algorithm has the following two steps:

(1) The first step is to search data cases, i.e., values of the node $\mathbf{C n}$, in the database, whose probabilitis are not zeros based on the sensing event obtained from the just-sensed corridor ${ }^{2}$. That is, the system stores the node Cn's values, which satisfy the following condition, in a list $\mathbf{c n l}=\left(c n l_{1}, c n l_{2}, \ldots\right)$.

$$
P(\mathbf{C n} \mid \text { obtained sensing information }) \neq 0
$$

Based on the results, we can estimate which data case in the recorded sensing information database is closer to the obtained sensing information.

(2) The second step is to predict possible actions $(P A)$ and sensor information $(S I)^{3}$ based on the obtained sensing information $(O S I)$ and the estimated $\mathbf{C n}$ values. The prediction is performed using the following probabilities:

$$
\begin{array}{r}
P(P A \mid \mathbf{c n l}, O S I)>t h d 2 \quad(a) \\
P(S I \mid P A, \mathbf{c n l}, O S I)>t h d 2 \quad(b)
\end{array}
$$

If the values of (a) and (b) exceed a certain threshold $t h d 2$, we save the possible actions in a list actlist, and save the predicted sensor information in a matrix $\mathbf{M}_{\mathbf{s e n}}$.

\section{Sensor Planning Procedure}

Through the prediction step, the system obtains actlist, a list of possible actions and also a matrix of predicted sensing information $\mathbf{M}_{\mathbf{s e n}}=\left(s n_{1}, s n_{2}, \ldots, s n_{n}\right)^{T}$, Each element of $\mathbf{M}_{\text {sen }}$ represents a predicted sensor information list to be obtained by a possible action (the element of actlist). Each predicted sensor information list of $\mathbf{M}_{\mathbf{s e n}}$ is sorted in the order of sensing cost, i.e. the distance from the current intersection to the location of the sensor information.

Consequently, the sensor planning process selects an optimal action from actlist which allows the robot to acquire enough sensing events to decrease ambiguity of the global localization belief by taking into account the trade-off between the sensing cost and the global localization belief.

For example, an actlist and a $\mathbf{M}_{\text {sen }}$ are shown in Fig. 5. The possible actions are "action1, action2, action3", and the integers on the right side of Fig. 5 represent expected sensor information to be obtained by the actions. In the Fig. 5, each row of the $\mathbf{M}_{\mathbf{s e n}}$ is one set of the predicted sensor information when taking the action on the left side. Every row of the $\mathbf{M}_{\mathbf{s e n}}$ is sorted in ascending order of the sensing cost, i.e., the sensing cost of the right entry is larger than that of the left. In the evaluation process, we use the elements of the $\mathbf{M}_{\text {sen }}$ and the possible actions to estimate the labels of the intersections, and calculate the sensing cost. Since the robot uses sensing information of a set of two neighboring corridors, the TolBef

\footnotetext{
${ }^{2}$ The prediction and planning processes are performed when the robot is in the middle intersection.

${ }^{3}$ It includes landmarks and intersection's geometric features expected to be perceived when the robot takes the actions
} 
should be defined as the sum of the maximum probabilities of the three intersection labels.

$$
\begin{aligned}
\text { TolBef }= & (1 / 3) \times(\max (P(\text { Head }))+\max (P(\text { Mid })) \\
& +\max (P(\text { Tail })))
\end{aligned}
$$

Using the above possible actions and predicted sensor information, the system performs the sensor planning which has the following three steps:

(1) The first step is to use the already-obtained sensing information, the possible action, and sensing information to be obtained by the action, to infer (TolBef), the belief of the global localization. In this step, we must evaluate every action and every set of sensor information (every row of $\mathbf{M}_{\text {sen }}$ in Fig. 5). For example, when we evaluate "action1" of Fig. 5 and the corresponding sensor information of the three rows, the procedures are as follows $((\mathrm{a}) \sim(\mathrm{d}))$ :

a) The system creates an empty list (SenEvn), and pushes the left-most element of the first row's sensor information into SenEvn.

b) Using the SenEvn, "action1" and obtained sensor information to estimate the TolBef based on Eq.2 and $B N$.

c)

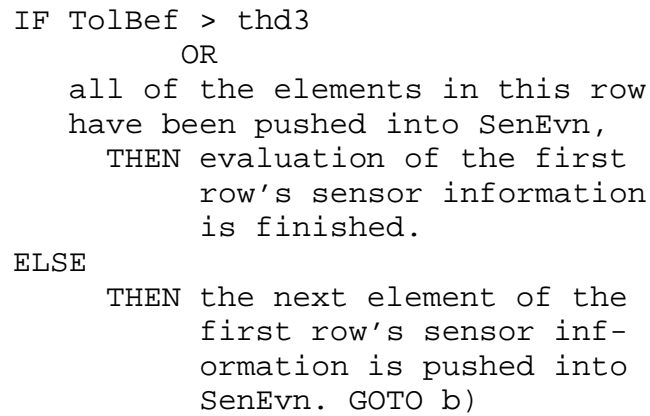

END IF

d) Using the procedure (a) (c), the system also evaluates the other two row's sensor information corresponding to "action1". After the above procedures are finished, the number of sensor information sets (count), which have beliefs of the localization TolBef $>t h d 3^{4}$ will be recorded. (count) represents to what degree the robot could determine the location when it takes that action.

(2) The system sums up the sensing cost of every row's sensor information, which is used in the first step (Cost), and also sums up the Cost of each row (sensing information sets) which satisfy TolBef $>$ thd3.

(3) Selects an optimal action by an efficiency criterion, i.e., an action which has the largest count and lower sensing cost.

For example, in the Fig. 5, each count of "action1" and "action3" is " 3 ", and the count of "action2" is " 1 ", so the optimal action can be selected from "action1" and "action3". Since the sensing cost of "action3" is lower than that of "action1", in this case, the optimal action should be "action3".

\section{E. Speedup of the Sensor Planning}

If the algorithm enumerates and checks all possible cases, enormous computation will be required in step (1) of the

\footnotetext{
${ }^{4}$ Since $T$ olBef $>$ thd 3 means the robot can uniquely determine three intersections' labels (entrance, middle, and exit intersection), in other words, the robot can determine its global location by "action1" and the first row's sensor information.
}

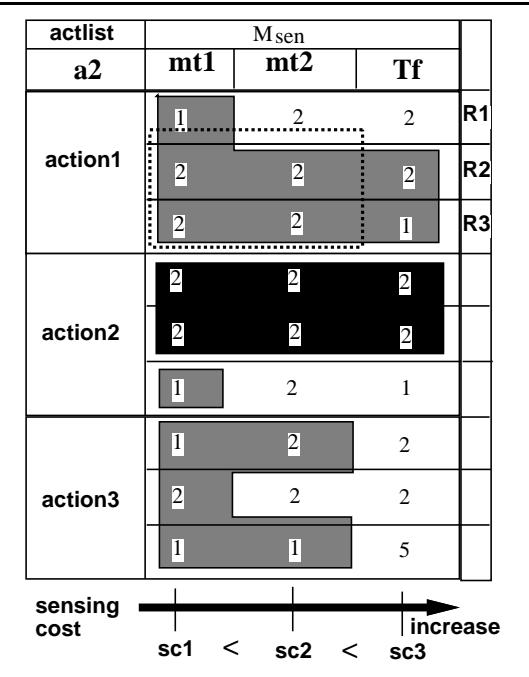

Fig. 5. An optimal action is determined by comparing the sensing information's local distance and geometric feature. The integer represents the sensing information. The numbers from left to right are values of $m_{t 1}, m_{t 2}$, and $T f$, respectively. Action 1 , action 2 , action 3 are values of $a_{2}$.

sensor planning. To reduce the computational cost, instead of simply running the $B N$ inference engine to evaluate the action and sensor information, we compare the sensor information features (include local distance and geometric feature) and check whether they can uniquely determine a location by the same action.

We will explain the method using the case of Fig. 5.

(i) We compare the sensing information of the $\mathbf{M}_{\mathbf{s e n}}$ that has the same action from the left side to right side. For example, the action l corresponds to three sets of sensing information. In the first row of the sensing information sets (the row with $R 1$ of Fig. 5), since the first element of rows $R 2$ and $R 3$ are " 2 ", if we get only the first sensing information " 1 " of row $R 1$, the system can distinguish the sensor information (row $R 1$ ) from the other two sets of sensor information (rows $R 2$ and $R 3$ ) which have the same action (action1). Of course, we can also use more sensing information of row $R 1$, but taking into account the sensing cost, using only the first sensing information, " 1 ", is more efficient.

(ii) We must test the TolBef(by Eq.2) using the selected sensing information $\left(" 1^{\prime \prime}\right)$ and its action, "action1". If TolBef $>t h d 3$, we consider that the first sensing information $\left(" 1^{\prime \prime}\right)$ of row $R 1$ expected by the "action1" is sufficient to uniquely determine a location. Otherwise, we must extend sensing information from its right side ${ }^{5}$ and test the TolBef until the condition TolBef $>t h d 3$ is satisfied.

(iii) Using steps (i) and (ii), we obtain the narrowest sensing range to distinguish the other sensing information sets which is shown in the gray region (before testing the TolBef) with the same action. If there are some sensing information sets, corresponding to the same action, which are identical, we cannot distinguish the information sets and cannot determine a location uniquely as shown in Fig. 5 by the black region.

\footnotetext{
${ }^{5}$ For example, in row $R 1$, if $"{ }^{\prime \prime}$ is not sufficient, we must add the right side of the elements, " 2 " or " 2,2 "
} 
